\title{
COMBINATORIAL OPTIMIZATION, COMBINED ALGORITHMS AND PROBLEM POSITIONING RADIO STATIONS BASED WIRELESS AND MOBILE
}

\author{
$\underline{\text { Aldemir M. de Oliveira }}^{1}$, Nelson Maculan ${ }^{2}$ \\ Federal University of Rio de Janeiro-UFRJ - PESC-COPPE \\ 1-PhD Student Program in Systems Engineering and Computing - PESC-COPPE / FUCAPI. \\ 2 - Federal University of Rio de Janeiro-Advisor \\ Ilha do Fundão-RJ-Rio de Janeiro-Brazil-Caixa Postal 68511 \\ $\underline{\text { amoliveira@gmail.com }^{1}}, \underline{\text { nelsonmaculan@gmail.com }}{ }^{2}$
}

\begin{abstract}
This article addresses the problem of positioning of base stations (BTS) in mobile telephony and specific points of a region (city) using the cell multipoint coordinated and Delaunay triangulation (CoMP-D) via genetic algorithm combined with Wolfpack. The goal is to meet or cover the largest amount of demand points using a minimum number of antennas. Are considered restrictions transmission range (target) and the presence of interfering obstacles (forest region).

To try to solve this combinatorial optimization problem primarily use the Law of Sines to determine the most appropriate angle in terms of sectorization of omnidirectional antennas and confront the results via simulation using the software that deals with the Wolfpack location using geometry angles via triangulation.

In a second stage of the research uses the Voronoi diagram and its dual, the Delaunay triangulation by applying the genetic algorithm to optimize the mobile network and mobile. To verify the good performance of the proposal we use the mobile phone network and the city of Manaus in northern Brazil and proves that the cell multipoint coordinated and Delaunay triangulation (CoMP-D) as a reliable approach treated the perspectives and leading to alternative interpretations and optimized solutions.
\end{abstract}

Keywords: Combinatorial Optimization, Algorithm Combined, Mobile Phones and Cell Phones, Delaunay Triangulation, Coordinated Multipoint. 


\section{1-Introduction}

Facility location problems deal with decisions about where to allocate or install facilities considering a demand that must be met in order to optimize one or more criteria. While the term "facilities" is used to denote health centers, warehouses, schools, factories, telecommunication antennas among others, while "clients" refers to neighborhoods, sales units, students etc. When we address these problems finding naturally contemplate their combinatorial nature, as they comprise selecting a discrete and finite set of data highlighting the best subset that meets certain criteria. Many of these combinatorial optimization problems are considered highly complex computational point of view. In general, heuristics are used for a "good" approximation of the solutions of the problems. Often these approaches are sufficient for the purpose of the user.

The literature suggests several formulations or models of localization problems [1]. The difference between these problems is in the way the applicants for the service and the location of these services is represented. The distance-applicant facility is an important difference between the models for the location of facilities. In some trouble location as known maximum distance, for example, a distance is given as the coating starting point [17]. To name a few we have expected problems covering sets, maximum coverage and maximum coverage

The maximum coverage problem in this research is to minimize the number of antennas for mobile telephony and mobile through a number of candidates for local facilities, this is, allowing that not every applicant for service is met. On the problems of coverage, the applicants are usually called the nearest facilities. Thus, it seeks to cover (meet) the applicant if it is within a given distance of the facility, and inefficient when the distance exceeds the amount considered or stated. In this context we mention the p-median problems, problems fixed costs and so on.

Several techniques have been applied to problems such as facility location, this problem has recently received considerable attention from academia. Several proposals using simulated annealing, genetic algorithm, tabu search, grasp, Lagrangean among others, are being used to solve this class of combinatorial optimization problems. This search will be guided by the following formula for this class of problem.

\section{1 - Defining the problem of locating Radio Base Stations ( RBS )}

Shows the definition of the problem of locating antennas, which subsidizes this work .

Notations:

$\mathrm{B}=\{1, \ldots, \mathrm{n}\}$ : set of demand points (a point of demand may be a neighborhood or a neighborhood block );

$\mathrm{A}=\{1, \ldots, \mathrm{m}\}$ : set of potential points where they can be located antennas or facilities (if the point $\mathrm{j} \in \mathrm{A}$ is located an antenna, then it is said that the facility $\mathrm{j}$ is open, otherwise the facility $\mathrm{j}$ is closed ); $\mathrm{Cj}$ : cost to open facility $\mathrm{j} ; \mathrm{d}$ : transmission range (radius of action ) of a radio base station or facility ; dij: distance (Euclidean) point $i$ to point $j ; a_{j}, b_{i}:$ variable decision $\in\{0,1\}$. If the user has already opens up $j=1, j=0$ otherwise. $b_{i}=1$ if demand point $i \in B$ is served by an open, otherwise $b_{i}=0$ ease; $N_{i}=\left\{j \in A \mid d_{i j} \leq d\right\}$ : set of facilities that can meet the demand point i .

\section{Formulation:}

\section{Maximize}

$$
\begin{aligned}
& f(x)=K \sum_{i=1}^{n} b_{i}-\sum_{j=1}^{m} C_{j} a_{j}-\sum_{i=1}^{n} \min \left\{d_{i j} \mid J \in A, a_{j}=1\right\} \\
& \text { s.a } \\
& \quad \sum_{J \in N_{i}} a_{j} \geq b_{i}, i=1, \ldots, n \\
& \quad \sum_{j=1}^{m} a_{j} \geq 1 \\
& \quad b_{i} \in\{0,1\}, i=1, \ldots, n \\
& \quad a_{j} \in\{0,1\}, J=1, \ldots, m
\end{aligned}
$$

The objective function (1) $\mathrm{f}: \theta \rightarrow \mathrm{R}$ ( $\theta=$ set of feasible solutions, $\mathrm{R}=$ set of real numbers) seeks to maximize the coverage (or care) of demand points using a minimum number of antennas and installing the antennas close as possible points of demand. $\mathrm{K}$ is a weight that prioritizes the the coverage demand points such that the objective function is always positive. Constraints (2) 
indicate that the demand points will be deemed covered (or attended) if they are within the coverage radius of an open facility (antenna). Constraint (3) ensures that at least should be open a facility.If an interfering obstacle exists between an open facility $\mathrm{j} \in \mathrm{A}$, and a demand point $\mathrm{i} \in$ $\mathrm{B}$, it is considered dij $=1000 \mathrm{~d}$. Depending on the geography of a city, there may be dense obstacles such as mountains, forests or hills that obstruct the direct target of the telecommunications signal [18].

Problems locating base stations (cell sites) occur quite frequently in the various segments of telecommunications, especially among which we can mention: providers, Internet access, signal transmission of radio, television, pager 's, repeaters and etc. A proper allocation of radio transmission equipment, including antennas is considered vital for the efficient service of the applicants, besides providing a considerable cost reduction for companies that offer the services. In the literature, the problem of locating antennas has been approached through the p-median model in which is fixed the number of antennas to be installed and are not considered interfering obstacles in range of the antennas [8] . Besides having a problem with high computational effort, the issue of growth steeply for services in mobile telephony and mobile corroborates this problem.

This growth in demand exponentially in the mobile phone in the world and has made the system evolves from the analogue to digital in a short time. According to the ITU (The International Telecommunication Union) there are 6.8 billion mobile subscribers worldwide, representing $96 \%$ of the world population. In developed and in terms of world countries, these subscribers are close to reaching the saturation level. This saturation is a consequence of mobile penetration in countries like China and India, the most populous countries in the world [2]. Moving to the side of developing countries this penetration is $89 \%$ of the population, according to ITU. At the end of 2012, there were 5.2 billion mobile subscribers in developing countries , accounting for $76.6 \%$ in terms of total subscriptions. According to the ITU, the forecast mobile subscribers worldwide will reach 7.0 billion by the end of 2013, 7.5 billion by the end of 2014 to 8.5 billion by the end of 2016.According to the above data, there is a continuous search for mobile service and mobile around the world that is guided by the need of the applicant in wanting increasingly send and receive data and require services with quality and speed to enable them. Therefore, it is within this context that the mobile phone comes ordering system and new approaches to the design of the physical layer and multiple access technologies, as well as the control of the radio link, in emerging systems, said fourth-generation or 4G [2], [3] and [ 4] . Given this approach, the 3rd. Generation Partnership Project (3GPP) believes in evolution of cellular networks such as LTE (Long Term Evolution) and LTE -A (Long Term Evolution Advanced) which use technologies such as orthogonal frequency division multiplexing (OFDM), multiple input multiple output (MIMO) and coordinated multipoint (CoMP), which in turn makes the mobile and cellular more complex and difficult to be modeled.

This paper applies the geometric theory of locating points on a plan proposed by the Ukrainian Voronoi and its dual, the Delaunay triangulation that has been used with great contributions when regard is to relate facilities facilities. The layout caused by the points in the plan allows a subdivision of that plan into sub-regions known in the literature by Voronoi diagrams. These diagrams provide a set of close relationships, for example, locating a company near your competitors and close to supermarkets or their respective customers proving its wide applicability [7]. Figure 1 below illustrates the Voronoi diagram and its dual to the city of Manaus.

Figure 1: Voronoi diagram and its dual to the city of Manaus.

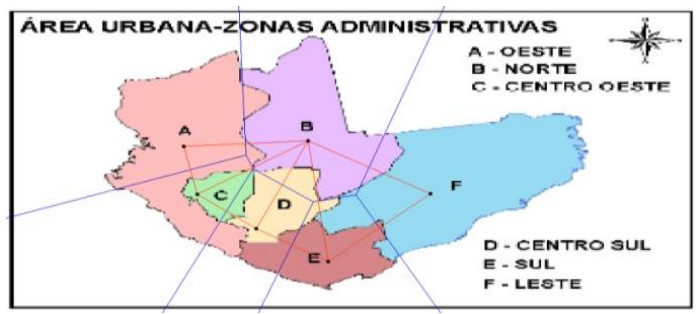


Applying the dual of the Voronoi diagram in mobile telecommunications and mobile, we can consider these points as RBS (radio base stations) and diagrams as coverage regions, thus allowing the division of space due to the connection to the nearest antenna and this so enjoy the close relationship provided by this theory. As demand for the service takes place in a shared manner prior knowledge of the multiple access technique is required, which enables different users to access a common channel simultaneously (or nearly so).

Multiple access is a technique that many subscribers or local stations can share the use of a communication channel simultaneously, or nearly simultaneously, despite the electromagnetic waves emitted by these stations may originate from widely different locations.

Building on the many successful applications of the Voronoi diagram and its dual Delaunay triangulation is believed that: The application of coordinated multipoint cell and called Delaunay triangulation (CoMP-D) problems of mobile cellular networks and may provide an alternative and efficient evolution interpretation compared to classical approaches.

\subsection{The Problem}

The problem of locating points taken as the starting point at each vertex of the Delaunay triangulation and its subgraphs (Relative Neighborhood Graph, Graph and Gabriel Directed Graph ) and the realization of the communication link has emerged during the literature survey in question. Have the CoMP - D cell can not be related to any of the featured topics, leading to the creation of a specific application group . Therefore, assessing the communication link and its coverage area through sectoral angle and basic property of the Delaunay triangulation with targeted and their subgraphs when obstacles arise in the communication link is required.

Departing for the first application, makes use of the Law of sines to verify the geometry of triangulation with respect to the area of the triangle and circle that circumscribes presented with various scenarios envisaged in $3 \mathrm{~km}, 4 \mathrm{~km}, 5 \mathrm{~km}, 10 \mathrm{~km}$ and $20 \mathrm{~km}$.

In the second subproblem makes use of RATM (relocation of antennas in the mobile telephone network and cellular) algorithm which is based on the FLIP algorithm when using a smaller amount BTSs 50 and genetic algorithm 50 for larger quantities. The RATM enables the relocation of the antennas (RBS) is charging point through the Delaunay triangulation which the new location of cell sites in the system and thus present a new scenario of coverage with respect to the areas of mobile network coverage and mobile.

This article is divided into five topics. The first presents the introduction, the problem . The second lecture on the theory of multipoint coordinated cell, the third topic simulation through genetic algorithm. The fourth topic our conclusions and future work.

\section{2 - Cell Coordinated Multipoint (CoMP)}

The CoMP (Coordinated Multipoint) system is a promising technology to increase the throughput of the system and allow an efficient allocation of radio resources, which can reduce the transmission power [9].

A CoMP system consists of a set of Radio Base Stations (RBS) geographically distributed and connected through a Base Station Radio Central (ERBC), where the amount of points the remote access system depends on several factors, one of which one is the system load, represented by the number of user equipment (UE) (User equipment) [8].

From Figure 2, it is possible to observe a CoMP system with a ERBC the center.

\section{Figure 2: A CoMP system with a ERBC.}

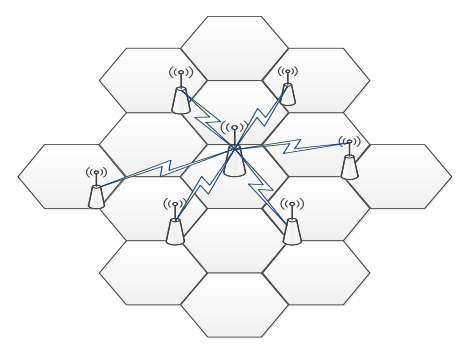


Starting from the theory proposed by Voronoi and its dual, we should check the quality of the resulting triangular mesh. And one of the points in relation to the submetering angulation in the mobile network base stations and mobile. And the natural question arises what is the angle that maximizes the coverage in the Delaunay triangular mesh? In response we use the law of sines to angles of 45, 60, 65,70 and 75 degrees and covered between stations 3, 4, 5, 10 and $20 \mathrm{Km}$.

The law of sines states that any triangle $\mathrm{ABC}$, the measures of the sides are proportional to the sine of the opposing angles and the extent of the opposite side divided by the sine of the opposite angle is constant and equal to $2 \mathrm{R}$, where $\mathrm{R}$ is the radius of the circle circumscribed to the triangle considered :

$$
\mathbf{a} /(\sin \mathbf{A})=\mathbf{b} /(\sin \mathbf{B})=\mathrm{c} /(\sin \mathrm{C})=\mathbf{2 R}
$$

Therefore, we will determine the radius of coverage of the circle circumscribing the triangle of CoMP - D hypothetical cell as is shown for the various explicit scenarios in Tables 1 below. This table presents a scenario at a time over the distance of sight between the RBS and the triangulation between the sides with their respective angles already described above.

\section{Table 1: Distances and angles referred to in CoMP-D hypothetical cell.}

\begin{tabular}{|c|c|c|c|}
\hline $\begin{array}{l}\text { Angle of the sector in the ERB } \\
\text { (Scenarios) }\end{array}$ & $\begin{array}{l}\text { Cell CoMP-D } \\
\text { (angle between the edges of } \\
\text { the triangulation) }\end{array}$ & $\begin{array}{l}\text { Distance of sight between } \\
\text { the ERB in } \mathbf{K m}\end{array}$ & $\begin{array}{l}\text { Scope of the radius of the } \\
\text { circle circumscribing the } \\
\text { triangle in } \mathrm{Km}\end{array}$ \\
\hline Scenario 1 & 45 degrees & 3 & 2,1285 \\
\hline Scenario 2 & 60 degrees & 3 & 1,7320 \\
\hline Scenario 3 & 65 degrees & 3 & 1,6559 \\
\hline Scenario 4 & 70 degrees & 3 & 1,5965 \\
\hline Scenario 5 & 75 degrees & 3 & 1,5529 \\
\hline Scenario 1 & 45 degrees & 4 & 2,8284 \\
\hline Scenario 2 & 60 degrees & 4 & 2,3094 \\
\hline Scenario 3 & 65 degrees & 4 & 2,2067 \\
\hline Scenario 4 & 70 degrees & 4 & 2,1285 \\
\hline Scenario 5 & 75 degrees & 4 & 2,0706 \\
\hline Scenario 1 & 45 degrees & 5 & 3,5355 \\
\hline Scenario 2 & 60 degrees & 5 & 2,8868 \\
\hline Scenario 3 & 65 degrees & 5 & 2,7584 \\
\hline Scenario 4 & 70 degrees & 5 & 2,6607 \\
\hline Scenario 5 & 75 degrees & 5 & 2,5882 \\
\hline Scenario 1 & 45 degrees & 10 & 7,0711 \\
\hline Scenario 2 & 60 degrees & 10 & 5,7736 \\
\hline Scenario 3 & 65 degrees & 10 & 5,5169 \\
\hline Scenario 4 & 70 degrees & 10 & 5,3214 \\
\hline Scenario 5 & 75 degrees & 10 & 5,1761 \\
\hline Scenario 1 & 45 degrees & 20 & 14,1422 \\
\hline Scenario 2 & 60 degrees & 20 & 11,5473 \\
\hline Scenario 3 & 65 degrees & 20 & 11,0338 \\
\hline Scenario 4 & 70 degrees & 20 & 10,6428 \\
\hline Scenario 5 & 75 degrees & 20 & 10,3530 \\
\hline
\end{tabular}

Elaboration of the autor

It is concluded that the scenarios presented through the various target considered in the table above, which as it maximizes the angle between the sectored antennas there is a fall within 
scope of the circle that circumscribes the triangle. However, considering the angle of 45 degrees in all scenarios would have a larger radius, on the other hand over a smaller angle. As we seek greater openness in order to maximize the coverage area, increasing the angle up to 75 degrees in the various scenarios was necessary and it was observed that the radius begins to decrease and it is concluded that the best angle corresponds to 65 degrees .

In the next section we will apply the genetic algorithm based on the verification of the Delaunay triangulation properties such as property of empty circle, a circle that denotes that circumscribes a triangle in the mesh or other triangle does not limit the assembly.

\section{3 - Simulation Based on Genetic Algorithm}

The algorithm starts with an arbitrary triangulation and becomes a Delaunay triangulation by changing the diagonals of the two neighboring triangles. These triangles share an illegal edge call, for not respecting the above property is then performed an operation called legalized or corner or edge sharing, which is illegal tip making this point a legal advantage, ie, the property that meets the circumcircle.

Taking the number of BTSs installed in the city of Manaus in 2013 according to data from Anatel will do a simulation using the same algorithm and confront with the output data as shown in Table 2 below.

Table 2: Statistics of the experiment after application of Genetic Algorithm.

\begin{tabular}{|c|c|c|c|c|c|}
\hline $\begin{array}{c}\text { Mobile } \\
\text { operators }\end{array}$ & RBSs & Experiment time & Solution & $\begin{array}{l}\text { Area larger than } \\
\text { the solution }\end{array}$ & $\begin{array}{l}\text { Smallest area that the } \\
\text { solution }\end{array}$ \\
\hline Claro & 121 & $\mathbf{8 2 7}$ & 105 & $\mathbf{2 7}$ & $\mathbf{2 9}$ \\
\hline Vivo & 205 & $\mathbf{1 2 7 9}$ & 116 & $\mathbf{6 2}$ & $\mathbf{6 7}$ \\
\hline Tim & 156 & $\mathbf{1 1 8 5}$ & 107 & $\mathbf{3 7}$ & $\mathbf{5 2}$ \\
\hline Oi & $\mathbf{1 0 9}$ & $\mathbf{8 1 1}$ & $\mathbf{9 4}$ & $\mathbf{1 3 8}$ & $\mathbf{3 2}$ \\
\hline Total & $\mathbf{5 9 1}$ & $\mathbf{2 1 8 4}$ & $\mathbf{3 2 1}$ & & \\
\hline
\end{tabular}

After watching all the screens comparing them since the beginning of the experiment arrived the following conclusions: The Claro with its 121 RBS arranged randomly and after implementation of the genetic algorithm solution showed 105 areas as relocation, areas larger than 27 solution and 29 lower areas that solution. The experiment time was 827/2. The operator Vivo with its 205 RBS had 117 solution areas, more than 62 solution areas, less than 67 solution areas. The time of this experiment was 1279 seconds. Tim operator with its 156 RBS 108 presented solution areas, the solution greater than 37 , less than 52 solution areas. The time spent was 1185/2. The operator Oi with its 109 RBS , 94 solution areas , 23 more than the solution, 32 less than the solution areas. The time of this experiment was 811 seconds. Taking all BTSs 591 this experiment showed 321 area as a solution, the solution greater than 138 areas less than 172 the solution areas. The time spent was 2184/2.

\section{4 - Conclusions and Future Work}

This article sought to investigate the possibility offered by multipoint coordinated cell and Delaunay triangulation as a hypothetical model in order to strengthen the relationship between communication points and speed in traffic. A bibliographical survey were detected several cases of successes in coordinated multipoint (CoMP) cell multiplexing input and output (MIMO) antennas related cooperation between the mobile cellular network. The success stories beheld the most diverse topics in mobile and cellular network such as antenna array design, network planning, prediction and propagation of radio frequency management and radio resource.

Supported by this line of research, this paper presents two proposals, each of which addressing a different problem.

The first problem relates to maximize the coverage after the location of cell sites. In this section, the law of sines was of great importance. It was found that the 65 degree angle provides for the compartmentalization maximizing the coverage in terms of the radius of coverage with the target. 
The second refers to the cooperation of the basic radio control (ERBC) on a network with multiple coordinate points and Delaunay triangulation (CoMP - D) based on genetic algorithm that makes the relocation of stations BTSs. The proper hypothesis of the problem enabled cell multipoint coordinated and Delaunay triangulation explore the problem from a perspective never before treated, providing an alternative analysis.

The benefits presented in terms of cooperation between transmission points and control were significant, such as relocating the radio base stations and the location of control stations on the threshold of the cells via Delaunay triangulation. Because when we locate one ERBC a vision of how many cell sites are near to cooperate between network points. However, one must consider that the indiscriminate growth of subsets with areas of large or small coverage and becomes unattractive when the number of UEs is not significant .

For both problems there are still many unexplored aspects that can be addressed in future research. In future research we applying metrics such as $\mathrm{L} 1$ and $\mathrm{L} \infty$, or insert repeaters for radio frequency signals to propagate the signal to remote communities in this region of continental size.

5 - References

[1] Drezner, Z. (1995). "Facility Location: A Survey of Applications and Methods" SpringerVerlag,New York, 1995.

[2] ITU - The International Telecommunication Union -2013.

[3] KHAN, A;QADEER, M; ANSARI.J; WAHEED, S.4G as a next generation wireless and communication, 2009. International Conference on 2009.

[4] KHAN,A; SUN, L; IFEACHOR, E. Learning models for vídeo quality prection over wireless local área network and universal mobile telecommunication system networks communication, IET - 2010.

[5] LAIHO, J.; RAIUIO, K; LEHTIRAAKI, P; HATONEN, K; SIMULA. $O$ advanced analysis methods for $3 G$ cellular networks wireless communications IEEE transactions on, v.4. ,n. $3,2005$.

[6] LATIF, I; KOLTENBERGER, F; KLOPP, R. LINK abstraction for multi-user MIMO in LTE using interference-aware receiver In. IEEE wireless communication and networking Conference, 2012.

[7] HAYKIN, Simon. Sistemas de Comunicação Analógicos e Digitais. 4 ed. - Bookman - São Paulo - 2004.

[8] Hoffmann, L.T e Gómez, A.T., (2003). Uma Abordagem do Problema de Localização de Torres de Rádio Transmissão Auxiliado por um Sistema de Informação Geográfica. XXXV $S B P O$, Natal.

[9] LIU, CHEN; Geirhofer. Downlink MIMO in LTE-advanced, IEEE, v.50, 2012.

[10] 3GPP TR 36.913, V9.0.0, "Requirements for Further Advancements for Evolved Universal Terrestrial Radio Acces 3GPP TS 36.300, V8.9.0. 2010.

[11] GESBERT, D. Hanly, S.;Shirts, S. Simeone; Yu, W. Mult-cell MIMO Coopeartive Networks. A New Look at Interference. IEEE jornal on Selected Areas in Communication V.28 p.1380-1408, Dezembro de 2010.

[12] OLIVEIRA, A. M; Nelson Maculan e Rosiane de Freitas. O Mercado de Telefonia Móvel Celular na Amazônia; Uma Abordagem em PLI - CMAC NORTE 2012 - Anais do Congresso de Matemática Aplicada e Computacional - ISSN -2317-3289.

[13] OLIVEIRA, A.M; Nelson Maculan; Rosiane de Freitas e Bruno Dias. Otimização Combinatória e o Problema da Alocação de Canais em Redes de Telefonia Celular-CMAC Nordeste 2012- Anais do Congresso de Matemática Aplicada e Computacional -ISSN -23173297.

[14] OLIVEIRA, A. M; Nelson Maculan; Bruno R. Dias; Rosiane de Freitas. Global Optimization Workshop 2012 - Proceedings of GOW, pp. 117 a 120. 2012.

[15] TOREGAS, C., Swain, R., Revelle, C., and Bergm, L., (1971). The Location of emergency servisse facilities, Operations Research, Vol. 19, pp. 1363-1373.

[18] SMIT, J. (1997). "Rádio Propagação", Editora Érica, $4^{\mathrm{a}}$ edição. 